DK $674.031 .32: 582.623 .2: 661.728 .6$
$539.388 .8: 535.827 .2: 537.533 .35$

Casperson, Gerhard, Philipp, Burkart, Jacopian, Vazgen, und Hoyme, Erika

Zum Quellungsverhaiten von Normalholz- und Zugholzfasern der Pappel

Faserforsch. u. Textiltechnik 20 (1969) 2, S. 61-70.17 Abb., 3 Tab., 35 Lit.

Das Quellungsverhalten von Zugholz- und Normalholzfasern von Populus robusta wird im fertig aufgeschlossenen, gebleichten, lufttrockenen Sulfitzellstoff mit lichtmikroskopischen sowie mit elektronenmikroskopischen (Abdruckverfahren, Ultradünnschnittverfahren) Methoden untersucht, wobei die Quelimittel Phosphorsăure, Cuen, Cadoxen und EWNN verglichen werden. Die Ursachen der im fertigen Zellstoff sehr ausgeprägten
Quellungsresistenz von Zugholzfasern werden diskutiert.
DK 677.494.745.32:677.494.061.3:[541.183:546.15]:539.26

von Hornuff, Günther, und Lenzer, Manfred

Einwirkung von Jod auf Polyacrylnitrilfaserstoffe

Faserforseh. u. Textiltechnik 20 (1969) 2, S. 86-98. 18 Abb., 8 Tab., 24 Lit.

Die Einwirkung des Jods auf die PVY-Faserstoffe wurde über das bei der Jodsorptionsmethode übliche Maß durch Erhöhung der Temperatur und Einwraungszeit intensiviert. An den jodierten PVY-Fasern wurden Struk列 fung sowie zu einer Verringerung der Reißfestiokeit, der Ori, der Schrumpdes Ordnungsgrades der PVY-Fasern. Die durch Jodbehandlung hervordes Ordnungsgrades der PVY-Fasern. Die durch Jodbehandlung hervorder Carrierwirkung geeignet.
DK 547.458.82:541.24.08:541.127.1:53.088.2

Poller, Siegfried

Zur Methodik der Polymerisationsgradbestimmung an Cellulosenitraten

Teil II. Geschwindigkeitsgefälle, Konzentration, Substitutionsgrad, Polymerisationsgradberechnung, Fehlerkritik

Faserforsch. u. Textiltechnik 20 (1969) 2, S. 71-76. 3 Abb., 6 Tab., 32 Lit.

Es werden t'berlegungen, Versuche und Berechnungen zur Methodik der Polymerisationsqradbestimmung an Cellulosenitraten angestellt. Im vorliegenden II. Teil der Arbeit werden die Probleme des Geschwindigkeitsgefălles, des Konzentrationseinflusses, des Substitutionsgrades und der
wertung der Viskositătszahlen in Polymerisationsgrade behandelt.
DK $677.463:[541.125: 543.888]: 66.094 .94$
$546.22 ; 546.33-36: 543.257 .1$

Pass, Heinz, und Michels, Christoph

Beitrag zur Bestimmung des Gammawertes im spinnenden Faserkabel

Faserforsch. u. Textiltechnik 20 (1969) 2, S. 77-80. 2 Abb. 2 Tab., 6 Lit.

Es wird von einer Methode der Gammawertbestimmung berichtet, bei der das mit einer speziellen Schneidvorrichtung aus einem spinnenden Faserkabel herausgeschnittene Probestück in kalter $2 \mathrm{~N}$ Natronlauge gelöst wird und nach Entfernung der Nebenprodukte durch Ionenaustausch die Ermittlung des in alkoholischer Natronlauge absorbierten Xanthogenatschwefels potentiometrisch erfolgt.

\section{DK $677.1 / .5: 531.754: 53.084$}

Winkler, Friedrich, Homann, Gerhard, und Götze, Wolfgang

Dichtebestimmung an Faserstoffen

III. Eigene Untersuchungen: Meßeinrichtung und Meßverfahren

Faserforsch. u. Textiltechnik 20 (1969) 2, S. $80-86.6$ Abb. 2 Tab., 5 Lit.

Die Entwicklung und der Aufbau einer Dichtemeßapparatur werden beschrieben, mit der die Dichte für die verschicdensten Faserstoffe nach dem Schwebeverfahren mit Konzentrationsvariation - Verfahren mit homogener Mrschung der Prufflussigkeit - bestimmt wurde. Der EinfluB der verfahrens wird angegeben.
DK $678.745 .32-492.2: 620.191 .7: 536.495$
$35.365 .1: 535.668 .8$

Makschin, Werner, und Ulbricht, Joachim

Untersuchungen zur thermischen Verfärbung des Polyacrylnitrils

Messung der Verfärbung und Vorrichtung \%ur Prüfung der thermischen Beständigkeit von Polyacrylnitrilpulvern

Faserforsch. u. Textiltechnik 20 (1969) 2, S. 98-101. 1 Abb., 3 Tab., 10 Lit.

Für die Ermittlung der thermischen Beständigkeit und der Verfărbung von Acrylnitrilpolymeren wird die Verwendung pulverförmiger Proben empfohlen. Eine Apparatur zur thermischen Behandlung von Polymerpulvern in einem beliebigen Gasmedium wird beschrieben, die vor allem die aus der schlechten Wärmeleitfähigkeit herrührenden Fehler eliminiert. Die Messung der Verfărbung erfolgt durch Bestimmung des Reflexionsgrades. Als Mabzahl wird die Große „prozentualer Verfarbungsgrad , das anderen $\mathrm{Meßgrößen} \mathrm{gegenūbergestellt.}$

Kurze Mitteilungen

DK 677.494:517.5:677.017.936

$535-31: 535-4: 539.412 .1$

Kaufmann, Siegfried

Treffertheoretische Analyse der Ergebnisse von Bestrahlungsversuchen mit ultravioletter und sichtbarer Strahlung Faserforseh. u. Textiltechnik 20 (1969) 2, S. 101-102. 1 Tab., 6 Lit.

\section{DK 661.728.63:674.031.12:539.388.8} $546.33-36: 541.12 .036$

Berndt, Wolfgang

Zur Quellung von Buchenzellstoffen in 19,5\%iger Natronlauge bei Temperaturen zwischen 20 und $70^{\circ} \mathrm{C}$

Faserforsch. u. Textiltechnik 20 (1969) 2, S. 102-103. 3 Abb., 4 Lit.

Neue Bücher S. 10/ -105 .

Patentschau S. $105-107$.

Literaturschau S. $107-110$. 


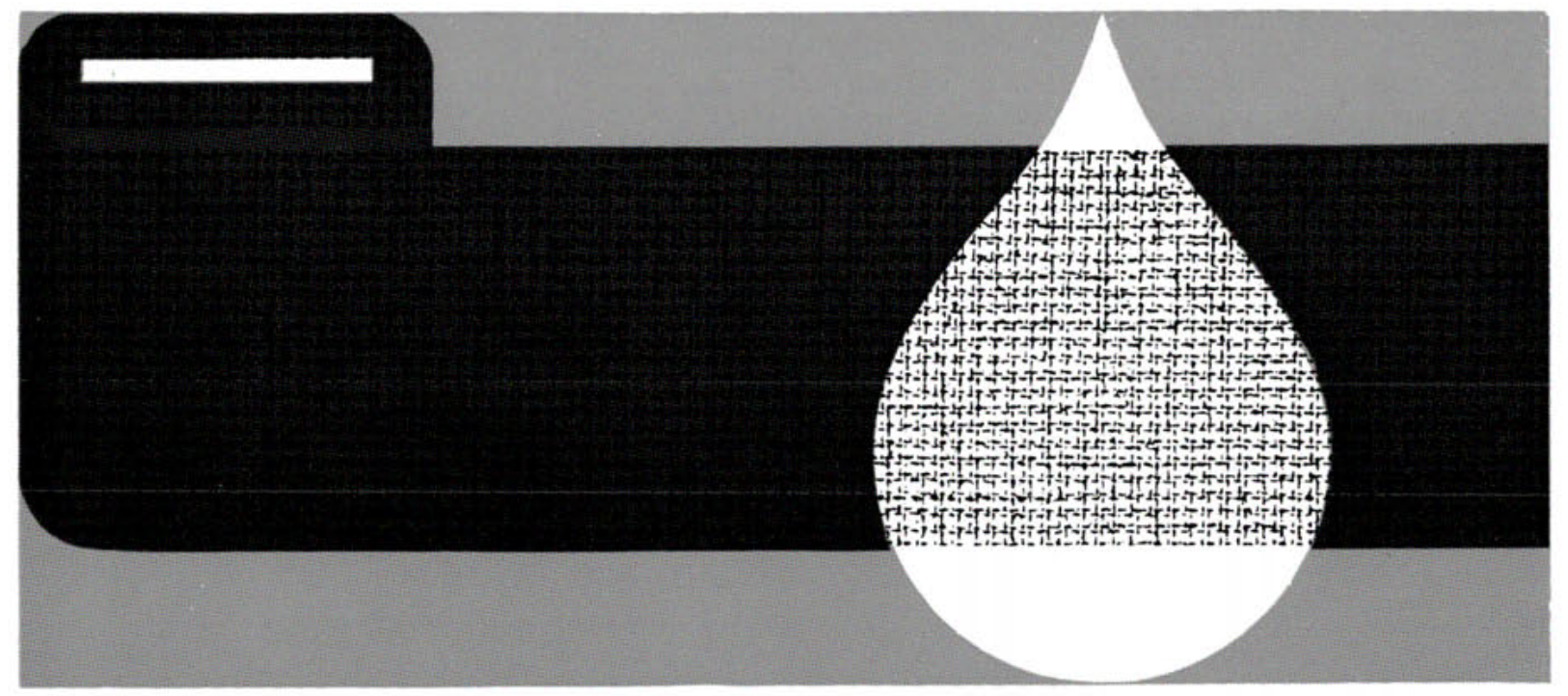

Fïr Pflegearm-Ausriistung:

React

Cerafil

Permalan

Antischmutz
Chemische Fabrik, 806 Dresden

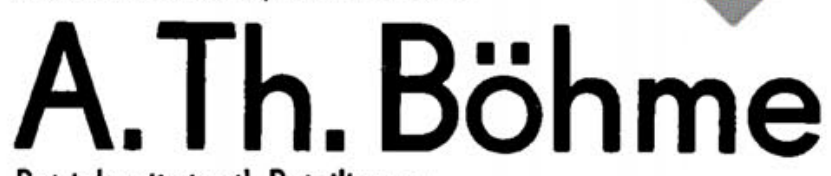

Betrieb mit staatl. Beteiligung
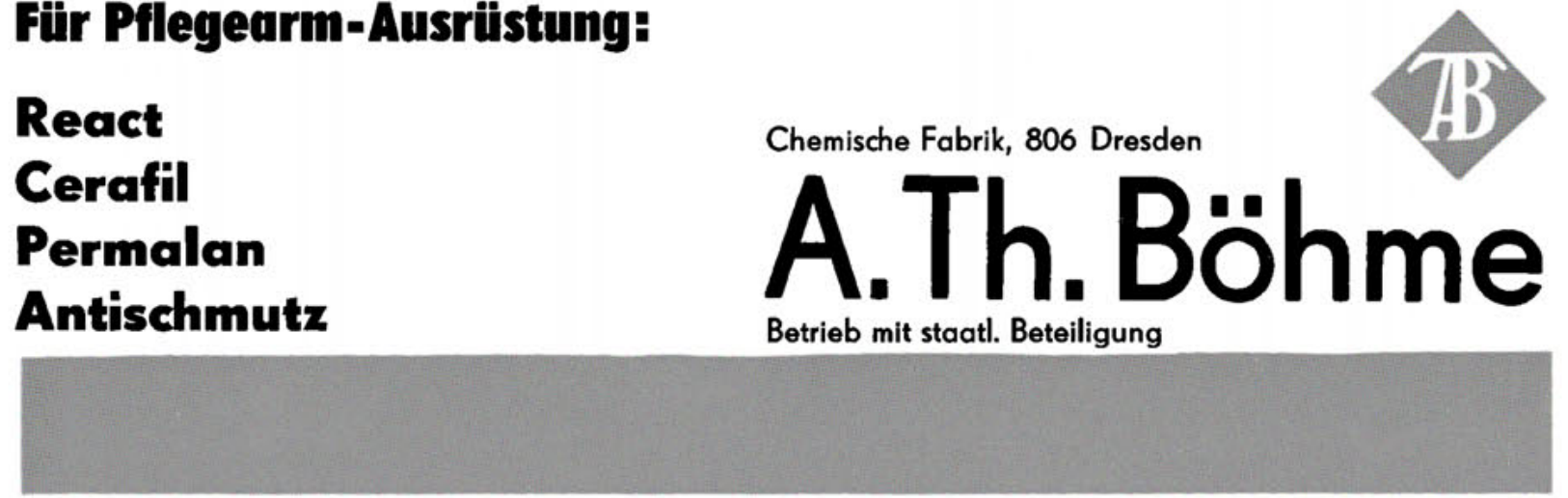

Die Zeitschrift „Faserforschung und Textiltechnik“ erscheint monatlich in Heften zu 48 Textseiten im Format A 4. Der Preis für das Einzelheft betrãgt M 15, - (Sonderpreis für DDR M 9,-), fur den Vierteljahrbezug M 45,- (Sonderpreis fúr DDR M 27, -), zuzügl. Bestellgeld. Die Berechnung erfolgt zu Beginn eines Vierteljahrs für 3 Hefte. Bestellungen aus dem Gebiet der Deutschen Demokratischen Republik an ein Postamt, eine Buchhandlung oder den Verlag, aus der Deutschen Bundesrepublik an eine Buchhandlung oder die Auslieferungsstelle Kunst und Wissen, Erich Bieber, 7 Stuttgart 1, Wilhelmstraße 4-6, aus dem Ausland an eine Importbuchhandlung, den Deutschen Buch-Export und -Import GmbH., 701 Leipzig, PostschlieBfach 276, oder den Akademie-Verlag GmbH., 108 Berlin, Leipziger Str. 3-4 (Fernruf: 220441; Telex-Nr. 011 2020; Postscheckkonto 35021) erbeten. Bestellnummer dieses

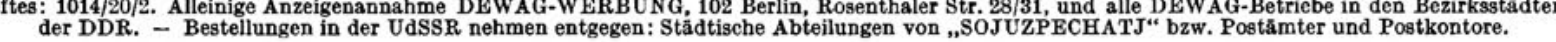

Herausgeber und verantwortlich für den Inhalt: Prof. Dr. Erich Correns, Institut für Faserstoff-Forschung der Deutschen Akademie der Wissenschaften zu Berlin, 153 Teltow-Seehof, Pernruf: Teltow 4831; Prof. Dr.-Ing. habil. Wolfgang Bobeth, Institut für Technologie der Fasern der Deutschen Akademie der Wissenschaften zu Berlin, 801 Dresden, Hohe Str. 6, Fernruf: 44721; Prof. Dr.-Ing. Hans Bőhringer, Institut für Textiltechnologie der Chemiefasern Rudolstadt, Fernruf: Rudolstadt 2031; Prof. Dr. Hermann Klare, Prof. Dr. habil. Burkart Philipp und Dr. Christian Ruscher, Institut für Faserstoff-Forschung der Deutschen Akademie der Wissenschaften zu Berlin, 153 Teltow-Seehof, Fernruf: Teltow 4831. Schriftleiter: Joachim Brämer und Dipl.-Chem. Ingeborg Ruscher, 153 Teltow-Seehof, Kantstr. 55. Verlag: Akademie-Verlag GmbH., 108 Berlin, Leipziger Str. 3-4. Satz und Druck: Druckhaus ,Maxim Gorki"
74 Altenburg. - Veróffentlicht unter der Lizenznummer 1280 des Presseamtes beim Vorsitzenden des Ministerrates der Deutschen Demokratischen Republik. Manuskriptsendungen sind an einen der Herausgeber oder die Schriftleitung zu richten. Für Inhalt und Form gelten die „Richtlinien für die Annahme und Abfassung von Beitrăgen", erhăltlich von der Schriftleitung. Die Verfasser gröBerer wissenschaftlicher Arbeiten erhalten außer dem Honorar ein Heft und 50 Sonderdrucke ihrer Arbeit unentgeltlich. Nachdrucke sowie tbersetzungen in fremde Sprachen des Inhalts dieser Zeitschrift und deren Verbreitung - auch auszugsweise mit Quellenangabe - bedürfen der schriftlichen Vereinbarung mit dem Verlag. 


\title{
Faserforschung und Textiltechnik
}

WISSENSCHAFTLICH-TECHNISCHE ZEITSCHRIFT FUR DIE CHEMIEFASER- UND TEXTILINDUSTRIE

\section{Zum Quellungsverhalten von Normalholz- und Zugholzfasern der Pappel}

\author{
Gerhard Casperson, Burkart Philipp, Vazgen Jacopian und Erika Hoyme
}

Deutsche Akademie der Wissenschaflen zu Berlin, Institut für Faserstoff-Forschung in T'eltow-Seehof

Das Quellungsverhalten von Zugholz- und Normalholzfasern von Populus robusta wird im fertig aufgeschlossenen, gebleichten, lufttrockenen Sulfitzellst off mit lichtmikroskopischen sowie mit elektronenmikroskopischen (Abdruckverfahren, Ultradünnschnittverfahren) Methoden untersucht, wobei die Quellmittel Phosphorsäure, Cuen, Cadoxen und EWNN verglichen werden. Die Ursachen der im fertigen Zellstoff sehr ausgeprägten Quellungsresistenz von Zugholzfasern werden diskutiert.

I6 поведению волокоп нормальной и тяговой древесины тополя при набухании

Методами световой и әлектронной микроскопии (методы получения реплик и ультратонких срезов) изучено поведение волокон тяговой и нормальной древесины Populus robusta после варки (сульфитный способ), отбелки и сушки при нормальной температуре. Проведено сравнение результатов, полученных при применении различных набухающих средств (фосфорная кислота, куоксен, кадоксен и жіВНК). Обсуждены причины ярко выраженной устойчивости к набуханию волокон тяговой древесины в готовой целлюлозе.

Swelling Behaviour of Normal and Tension Wood Fibres of Poplar (Populus robusta)

The swelling of normal and tension wood fibres in sulfite pulp of Populus robusta has bcen investigated by optical and electron microscopy (replicating, ultrathin sectioning) after pulping, bleaching and air-drying, using phosphoric acid, Cuen, Cadoxen and EWNN as swelling agents. The considerable resistance to swelling of tension wood fibres in the pulp produced is discussed.

\section{Einleitung}

Zur Charakterisierung von Holz- und Zellstoff-Fasern wird oft auch die Linwirkung von Quellmitteln herangezogen, wobei die Auswertung in der Regel lichtmikroskopisch erfolgt [1 bis 6]. Elektronenmikroskopisch wurden die verschiedenen Quellstadien bisher wenig untersucht, was zum Teil durch die Schwierigkeiten bei der Präparation gequollener Fasern bedingt sein mag [7]. Aufbauend auf frühere lichtmikroskopische Arbeiten von Haas [8], von Correns [9] und von Sachsse [10] \%ur Quellung von Zughol\%fasern der Pappel, soll im folgenden über licht- und elektronenmikroskopisehe Untersuchungen zum Quellverhalten vollständig aufgeschlossener und gebleichter ZellstulfFasern aus Pappelhol\% berichtet werden. Entsprechend der von uns bearbeiteten Gesamtthematik, $d . h$. einer Klärung des Reaktionshol\%-Einflusses auf Iterstellung und Verarbeitung von Pappeltextilzellstolf [11 bis 13] mittels chemischer und morphologischer $\Lambda$ rbeitsmethoden, steht dabei ein Vergleich von Normalholz- und Zughol\%faser im Vordergrund. Daneben soll mit dieser Arbeit ein Beitrag zur Frage des unterschiedlichen Quellverlaufs in verschiedenartigen Quell- und Lösemitteln für Cellulose gegeben werden.

\section{Bemerkungen zur l'ersuchsmethodik}

\subsection{Herstellung und Charakterisierun! der Zellstoffproben}

Zugholzreiche, friseh eingeschlagene und entrindete Stämme von Populus robusta (Standort Dippoldiswalde/ Sa.) wurden in ea. $3 \mathrm{~cm}$ dicke Scheiben zerlegt und diese nach Trocknen in I.uftumwälztrockenschrank bei $50^{\circ} \mathrm{C}$ in Blöckchen von ca. $30 \times 30 \times 5 \mathrm{~mm}$ Kantenlänge zerkleinert, worauf eine sorticrung in praktisch zugholzfreie
(,Normalholz") und besonders zugholzreiche (,Zugholz") Blöckchen vorgenommen wurde. Der Aufschluß erfolgte nach dem Calciumbisulfit-Verfahren mit $0,95 \% \mathrm{CaO}$ und $6 \%$ Gesamt- $\mathrm{SO}_{2}$ bei einem Flottenverhältnis 1:4,5. Anschließend wurde dreistufig gebleicht. Zugholz und Normalholz wurden entweder in getrennten Ansätzen aufgeschlossen (,separate Kochung"), oder aber in einer gemeinsamen Kochflotte, wobei Normalhol\%- und Zugholzcharge durch ein Drahtnetz im Siebeinsat\% des Autoklaven voneinander getrennt waren (,,gemeinsame Kochung"). Hinsichtlich methodischer Einzelheiten sei auf unsere kürzlich erschienene Veröffentlichung verwiesen [14].

Die wesentlichen Analysendaten der für die Quellungsuntersuehungen eingesetzten Proben gehen aus Tabelle 1 hervor, wobei die unterschiedlichen Kocherstoff-DP von Zugholz- und Normalholzcharge bei gemeinsamer Kochung in [14] bereits ausführlich diskutiert wurden.

\subsection{Durchführung der Quellungsuntersuchungen}

Als Quellmittel dienten 80\%ige Phosphorsäure, CuenLösung nach [16], Cadoxen-Lösung nach [17 bis 19] sowie EWNN [20], hergestellt nach [21]. Die alkalischen Quellmittel gelangten sowohl unverdünnt als auch in den später angegebenen Verdünnungen mit Wasser zur Anwendung.

Für die lichtmikroskopischen Untersuchungen wurde ein Nf-Forschungsmikroskop (VEB Carl Zeiss Jena) mit Phasenkontrast und Polarisationseinrichtung verwendet. Die Quellbilder der Einzelfasern wurden im verdünnten Quellmittel beobachtet und fotografisch aufgenommen. Zum Vergleich der Quellgeschwindigkeiten in EWNN, Cuen und Cadoxen wurde bei schwächerer Vergrößerung auf einem Objektträger mit lufttrockenem Probenmaterial ein Gesichtsfield mit ca. 50 Einzelfasern ausgesucht, dann von der 